\title{
Chasing red herrings: Memory of distractors causes fixation in creative problem solving
}

\author{
Zsolt Beda $^{1}$. Steven M. Smith ${ }^{1}$ \\ Published online: 7 March 2018 \\ (C) Psychonomic Society, Inc. 2018
}

\begin{abstract}
Two experiments tested the red herring retrieval hypothesis, which states that fixation in creative problem solving is worse when memory for red herrings (i.e., inappropriate or incorrect solutions) is strengthened. In Experiment 1, when associations between Remote Associates Test (RAT) problem words (e.g., COTTAGE, SWISS, CAKE) and related red herring words (e.g., hut, chocolate, icing) were strengthened via repetition, an encoding variable, fixation was found to increase. In Experiment 2, when associations were formed between RAT problem contexts and red herrings, then subsequent reinstatement of problem contexts during RAT problem solving trials (as compared with showing new contexts) also worsened fixation effects. Our results add to the increasing body of research that connects memory with creative problem solving, and they suggest possible ways for overcoming fixation effects.
\end{abstract}

Keywords Creativity · Remote Associates Test · Fixation · Context

A few years ago one of the authors spent some days in Florida in a small hotel on the beach. During one of his morning runs on the shore, he realized he had forgotten the name of his hotel. Having repeatedly stayed at another hotel on a similar Florida beach, the name of the previous hotel The Diplomat came to mind, blocking his memory of the correct hotel name. He only found out the name of his hotel when he got back from his run, it was The Prestige. This is an example of red herring retrieval, that is, a mental block that occurs when a wrong answer comes from memory, blocking the correct response. In this example, two factors influenced retrieval of the wrong answer: repetition (he had stayed at The Diplomat twice) and context (it was on a similar beach in Florida). In the present study we tested a laboratory version of red herring retrieval, examining the influence of two factors known to affect memory of such wrong answers' retrieval, repetition and context.

Exposure to wrong answers has been shown to impede creative problem solving (Smith \& Blankenship, 1989, 1991; Wood \& Pennington, 1973), a finding referred to as a

Zsolt Beda

bedazs@tamu.edu

1 Department of Psychological and Brain Sciences, Texas A\&M University, College Station, TX 77843, USA fixation effect (e.g., Kohn \& Smith, 2009; Smith, 1995a, b, 2003; Smith \& Blankenship, 1989, 1991; Wiley, 1998). Some studies of fixation effects have presented these wrong answers, what we will refer to as red herrings, simultaneously with problems (e.g., Smith \& Blankenship, 1989, 1991), others have pre-exposed participants to these misleading stimuli (e.g., Smith \& Blankenship, 1991; Wood and Pennington, 1973), suggesting that such fixation effects might be attributable to retrieval of the wrong answers. Do manipulations of variables that affect long term memory retrieval of red herrings affect observed levels of fixation in creative problem solving? Although it has been implied by previous studies that the memory strength of red herrings determines the strength of the subsequent fixation effect that occurs, in fact, there have been no previous reports of tests of this principle, what we will refer to as the red herring retrieval hypothesis.

We use the term red herring rather than blocker because the term blocker implies both a cognitive process, response competition, and a result, namely, poorer performance. The presentation of misleading or wrong answers to problems might, or might not cause the intended reduction in performance, and even if they do, they may decrease problem solving performance via a different cognitive mechanism than response competition. Here, we use the term red herring to indicate the intended effect of such stimuli, that is, to 
divert the problem solver from the path that leads to a correct solution, just as the use of red herrings was intended to divert hunting hounds from the trails of game animals.

In the creative cognition literature (e.g., Finke, Ward, \& Smith, 1992) many studies have examined fixation effects, that is, blocks to creative problem solving, which can be caused by various factors. For example, Duncker (1945) studied functional fixedness, that is, fixation based on the commonly known functions of experimental objects. In another example, Luchins and Luchins (1959) demonstrated the negative effects of mental set with their Einstellung paradigm. Smith and Blankenship (1989) experimentally induced fixation by presenting clue words intended as red herrings for creative problem solving; they found that presenting red herrings reduced initial performance on rebus problems. Although fixation can have many different causes, the experiments of Smith and Blankenship (1989) indicate that remembered red herrings can cause a fixation effect.

In addition to the experiments of Smith and Blankenship (1989), other studies have also used the method of preexposure to red herrings before participants attempt creative problem solving. Some studies that have used Remote Associates Test (RAT) problems (Mednick, 1962) with this pre-exposure technique include Smith and Blankenship (1991), Vul and Pashler (2007) and Wood and Pennington (1973).

Furthermore, there have also been studies that experimentally pre-exposed participants to examples of responses to a subsequent creative idea generation task (Smith, Ward, \& Schumacher, 1993; Landau \& Leynes, 2004), or a brainstorming task in a group (Kohn \& Smith, 2011). Such studies of conformity effects are similar to studies of fixation in creative problem solving because they show that participants are constrained to using features of the preexposed examples. If this happens in a group, initial ideas might even create a collaborative inhibition, which will impair the performance of the whole group working on the same problem (Weldon \& Bellinger, 1997). There is also a growing literature on design fixation effects (Jansson \& Smith, 1991; Chrysikou \& Weisberg, 2005; Purcell et al., 1993, Sio, Kotovsky, \& Cagan, 2015; Vasconcelos et al., 2017), which shows that fixation to design examples, or even to past design works can create constraints when facing a task requiring a novel design solution. Thus, there are constraining effects of experimentally controlled stimuli that can impede creative cognition across a range of creative activities.

In a general sense, the RAT fixation paradigm that involves pre-exposure to red herrings resembles negative transfer paradigms that have been studied at least since Müller and Pilzecker (1900). Negative transfer is said to occur when prior learning causes poorer subsequent performance, such as poorer interpolated learning in retroactive interference experiments (e.g., Neill \& Valdes, 1992). A lengthy body of literature exists that shows negative transfer using recall tests and other direct measures of memory, that is, tests in which the to-be-remembered events are explicitly referred to in the directions to participants. A smaller literature shows negative effects of prior learning on indirect measures of memory, that is, tests that do not refer to the events in question. Relative to direct measures of memory, such as cued recall, indirect measures, such as word fragment completion, anagram solution, or category production may involve different amounts of conscious recollection and automatic retrieval (Jacoby, 1998). Indirect memory measures can be affected, for example, by numbers of repetitions of target items (e.g., Jacoby \& Dallas, 1981), and by manipulations of perceptual modality between encoding and test (e.g., Kirsner, Milech, \& Standen, 1983; Scarborough, Gerard, \& Cortese, 1979). In the present study we ask whether RAT problem solving, a test typically used to assess creativity (e.g., Benedek \& Neubauer, 2013), can function as an indirect measure of memory. Given that RAT problems are solved by retrieving candidate associates to the test words, we assume that the RAT would be more of a conceptually-driven test, rather than a primarily data-driven one. Is RAT performance affected by encoding and retrieval manipulations (of red herrings), such as repetition and context reinstatement?

A negative transfer effect, with an indirect memory measure was demonstrated and studied by Smith and Tindell (1997), who used as blockers (or red herrings) words that were orthographically similar to word fragments that were subsequently tested. Smith and Tindell (1997) showed that this memory blocking effect with an indirect memory measure followed the predictions of the transfer appropriate processing theory; manipulations of level of processing at encoding did not influence the blocking effect seen on the primarily data-driven fragment completion test, but changes in perceptual modality (from auditory at encoding to visual at test) did influence the effect. Similar paradigms yielded similar results at Logan and Balota (2003), Landau and Leynes (2006), and Leynes, Brown, and Landau (2011). Lustig and Hasher (2001) reported similar findings in their study of implicit memory. Furthermore, Landau and Leynes (2006), who dubbed this finding the memory blocking effect (MBE), reported that neither time, nor depth of processing or awareness of the MBE affected the magnitude of the effect.

The other body of findings of negative transfer effects using an indirect memory measure come from exclusion tasks in the process dissociation procedure (e.g., Jacoby, Toth, \& Yonelinas, 1993; Jacoby, 1998). For example, after reading a list of words, a participant may be asked to complete word stems without using words recollected from the previously encoded study list (e.g., Jacoby, 1998), or 
completes a cue-word+stem with a related word not from the study list (e.g., Jacoby, 1996). In these opposition tasks, automatic retrieval of forbidden items can supplant correct responding, but only when recollection of the studied responses fails. In the context of the present study, one may think of the forbidden (studied) responses on exclusion tasks as similar to red herrings that lead participants away from correct responses.

If fixation in creative problem solving can be increased by making red herrings more retrievable, then we can think of creative problem solving as a type of indirect memory measure, and fixation caused by retrieval of red herrings as analogous to other types of negative transfer effects. Our red herring retrieval hypothesis states that factors that make red herrings more retrievable should reduce creative problem solving performance, as measured with RAT problems.

Several studies have shown that pre-exposure to various types of red herrings can cause subsequent fixation or conformity effects in creative problem solving, creative idea generation and memory retrieval, however none of these studies has provided a test of the red herring retrieval hypothesis, which states that the memory strengths of red herrings determine the magnitude of a fixation effect. Alternatively, strengthening the retrievability of a red herring might decrease fixation effects if faster retrieval of red herrings allows them to be rejected more quickly. The faster one can put aside a wrong answer, the more able one will be to devote more mental resources to searching for the correct answer. We conducted the present experiments to test the different possible outcomes of increasing retrievability of red herrings.

The present study In the present experiments a fixation effect was elicited using previously studied red herrings, to make it difficult for participants to solve Remote Associate Test (RAT) problems (Mednick, 1962). In the RAT, often used as a measure of creative ability (e.g., Benedek \& Neubauer, 2013), there are three test words in each problem (e.g., COTTAGE - SWISS - CAKE.) The task is to find a single word that can be used to make a compound word or a two word phrase with each of the three test words. The solution for this particular test item is CHEESE (COTTAGE CHEESE, SWISS CHEESE, CHEESECAKE.) To elicit a fixation effect, paired associates were assigned to each of the three test words. These paired associates were conceptually related to the test words, but were not related to the solution. For example, for the RAT problem COTTAGE - SWISS - CAKE the red herring associates made the pairs COTTAGE - hut, SWISS - chocolate, CAKE - icing. We predicted that when the red herrings are made easier to recall, the observed fixation effect would be greater.
Numerous factors can affect the accessibility of events, including encoding and test manipulations. In the present study, we tested our red herring retrieval hypothesis by manipulating an encoding variable: number of practice trials, and a test variable: context reinstatement. If the red herrings are repeated and practiced more, then more of them should be retrieved when a triad of RAT words are presented. Repetition improves both recollection and automatic retrieval of items in memory (e.g., Jacoby \& Dallas, 1981). Therefore, lower solution rates were expected when there were more repetitions and practice trials of red herring words, as compared with less practice. Similarly, the context manipulation was predicted to affect retrieval of red herrings on RAT trials: when the initial encoding context was reinstated, more red herrings were expected to be retrieved (e.g., Smith, 1979; Smith \& Vela, 2001; Smith \& Manzano, 2010; Smith et al., 2014), causing solution rates to suffer for those RAT problems. Several studies have reported context reinstatement effects with indirect memory measures (e.g., Parker, Gellatly, \& Waterman, 1999; Smith, Heath, \& Vella, 1990), but whether such effects are caused by automatic retrieval or conscious recollection may still be in question (see Mulligan, 2011; Smith et al., 2018). We expect to find context dependent fixation regardless of whether our manipulations of context affect conscious recollection, automatic retrieval, or both types of retrieval processes.

The method in the present experiments used a particularly robust paradigm for context-dependent memory effects, including the use of perceptually rich contexts (photo backgrounds of various environments), small context-to-item fans (only three words per context), and intentional encoding of items (red herrings, in our case) with their associated contexts (see Smith, 2013). Thus, we expected context reinstatement of red herrings, relative to non-reinstatement, to cause poorer performance on associated RAT problems (Experiments 1 and 2), and we expected that more practice with red herrings, relative to less practice, would cause poorer RAT solution rates (Experiment 1).

\section{Experiment 1}

Participants in Experiment 1 were given four tasks that were aimed at making sure that the photo contexts were well encoded (the context encoding task), that red herrings were associated with RAT test words (the paired associates encoding task), and that test word-red herring associations were strengthened (the retrieval practice task). After the red herrings, contexts, and RAT test words were encoded, the Remote Associates Test was given; each critical RAT triad was tested by being superimposed over a test context, which either reinstated the encoding contexts of red herrings, or presented a different context than the one seen for that triad 
at encoding. It was predicted that more practice with red herrings, relative to less practice, and context reinstatement at test, relative to non-reinstatement, would both decrease performance on critical RAT problems, because practice and context reinstatement should improve retrieval of red herrings. Our context reinstatement prediction, however, was predicated on the assumption that the three word-word associations created for each RAT triad would not outshine (or overpower) the weaker context-to-word associations (Smith \& Vela, 2001).

\section{Method}

\section{Participants}

Based on an a priori power analysis, our experiment needed approximately 72 participants to detect a medium sized effect (Cohen's $d=.5$ ) with $\alpha=.05$ and $1-\beta=$ .95. Participants were undergraduate psychology students volunteering as participants to fulfill a course requirement. They could enroll for the particular time slots via a computerized database. The sessions were conducted with 3-15 participants at a time. Altogether we had 133 participants with $30,32,35$ and 36 in four counterbalancing groups. The numbers of participants in the counterbalancing groups varied because participants could enroll themselves in any session of the experiment, and experiment sessions were randomly assigned to the counterbalancing conditions.

\section{Design and materials}

A total of 36 RAT problems were used in Experiment 1. A RAT problem consists of three words, and the solution is a single word that is strongly associated with each of the three test words (e.g., COTTAGE - SWISS - CAKE, solution - CHEESE). The problems were selected from the Compound Remote Associate Problem compendium published by Bowden and Jung-Beeman (2003), using problems with high normed levels of solution rates. For each triad of RAT words, red herrings were generated, one red herring word for each of the three test words, resulting in three paired associates for each RAT problem (e.g., COTTAGE-hut, SWISS-chocolate, CAKE-icing). Test words, solution words, and red herring words were unique within our set of materials; none were repeated in other problems. All of the RAT test words were presented in capital letters and all of the red herrings in lowercase letters throughout the experiment. RAT problems, their solutions and their associated red herrings are shown in the Appendix. The items were presented as a PowerPoint slideshow shown on a large screen in clear view of the participants.

A total of 72 photo contexts, or photographs of places, were used in Experiment 1. The photos showed familiar types of places, such as an airport or a restaurant, but the specific places were likely not known to our participants. Each photo context was assigned at random to a RAT problem, except that obvious pre-experimental associations of words and accompanying photos was avoided in each pairing. Stimulus words were shown in a red font and outlined with a yellow border, and they were superimposed over their accompanying photo contexts. On retrieval practice trials, the encoding contexts accompanying paired associates were the same contexts paired with those items at encoding. On the RAT, triads were tested either with the photo context of the red herrings' encoding, or with a new photo, that is, a photo not seen before in the experiment.

A 2 (reinstatement, reinstated or new contexts at the test phase, a within-subjects variable) $\times 2$ (repetition, zero or two retrieval practices, a within-subjects variable) $\times 4$ (counterbalancing, a between-subjects variable) mixed design was used. The 36 RAT triads were randomly divided into four sets of nine triads, sets A, B, C, and D. In the first counterbalancing group (CB-1), items from set A were used for the zero-practice new-context condition, set $\mathrm{B}$ was used for the two-practice new-context condition, set $\mathrm{C}$ for the zero-practice reinstated-context condition, and set $\mathrm{D}$ for the two-practice reinstated-context condition. In CB2, CB-3, and CB-4, each set of items was assigned to a different condition, so that across the four counterbalancing groups, each item was used in each treatment condition. The proportion of correctly solved RAT problems in the final phase of the experiment served as our dependent measure. Strict scoring was used, that is, no alternative solutions to the RAT problems were accepted.

\section{Procedure}

The PowerPoint slideshow had four sections corresponding to the experimental tasks, context encoding, paired associates encoding, paired associates retrieval practice, and finally, the Remote Associates Test. Participants were initially told that they would complete several different tasks during the experiment and that they would receive instructions when it was time for each task. Each task consisted of 36 slides, one for each of the critical items, that were presented in an order randomized in blocks of six.

For the first task, context encoding, participants gave relatedness ratings on a 1-10 scale between each set of three red herring words and their background context. The backgrounds were not conceptually related to the red herrings in any obvious way. Participants had 8-sec to look at the screen, and write down their ratings.

For the next task, paired-associates encoding, participants tried to memorize triads of paired-associates consisting of the three test words each linked with its red herring 
Context encoding

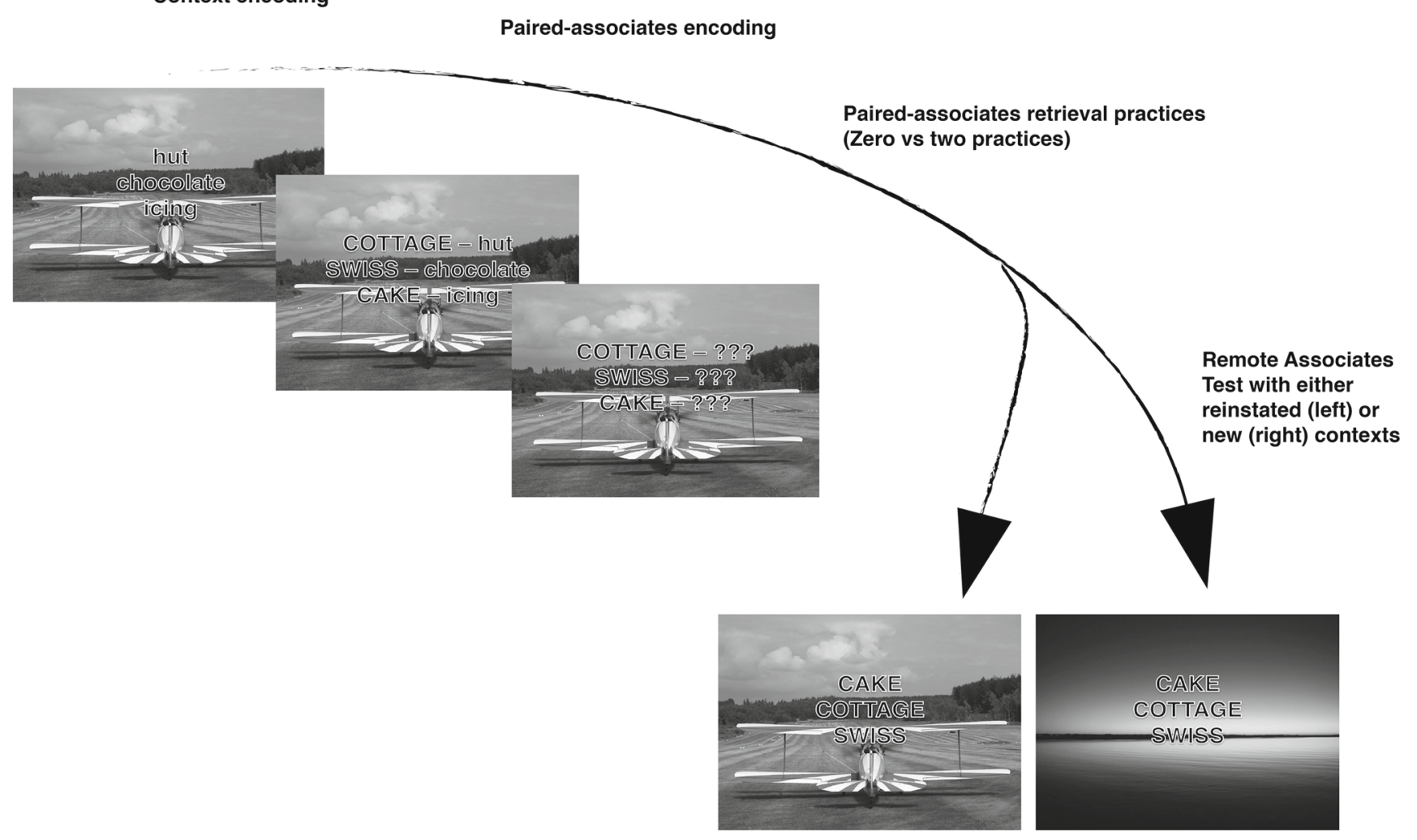

Fig. 1 Experiment 1 method

word. Each triad of paired-associates was superimposed over the same photo context that had been seen with the triad's red herring words at encoding. Each study trial of the paired-associates encoding task was 5-sec.

Following paired-associates encoding was the pairedassociates retrieval practice task. For half of the items, on each trial of the retrieval practice task, participants were shown the three RAT words superimposed over their encoding context (only for items in the two-retrieval practices condition), and they tried to recall and write down the three words that had been the responses (the red herrings.) Participants had 10 -sec for each practice trial. After 10-sec, the correct responses were displayed, and participants either circled their response if they got it right, or they wrote down the solutions they could not remember. This self-correction procedure took an additional 10-sec. There were two retrieval practice trials for each item in this condition; the two practice trials were evenly distributed, with at least 8-10 slides between the two trials.

The final task was the Remote Associates Test. For each problem, participants had 15 -sec to think of the solution. The word triads were superimposed over either the encoding background for the associated red herrings (reinstated context condition) or a different context that had not been seen previously in the experiment (new context condition.) The order of the three words was never the same on the
RAT task as it had been on the encoding or the retrieval practice tasks. No mention was made of the previous tasks in the instructions. The method of Experiment 1 is shown on Fig. 1.

\section{Results}

A 2 (context: reinstated, new context; a within-subjects variable) $\times 2$ (repetition: zero, two; a within-subjects variable) analysis of variance (ANOVA) was computed with the proportion of RAT problems solved as the dependent variable. ${ }^{1}$ The main effect of repetition was significant $\left[F(1,132)=5.167, p=.025\right.$, partial $\left.\eta^{2}=.038\right]$; items that had two retrieval practices with red herrings, relative to items with no practice trials, had lower solution rates, as predicted by the red herring retrieval hypothesis. The main effect of context was not significant $[F(1,132)<1$, $p=.541$, partial $\left.\eta^{2}=.003\right]$; although, numerically, the reinstated context condition was slightly worse than the new context condition, as predicted, the difference was small. The context $\mathrm{X}$ repetition interaction was not significant

\footnotetext{
${ }^{1}$ The inclusion of counterbalancing in a mixed ANOVA as a between-subjects measure showed no differences between the four counterbalancing groups $\left[F(3,129)<1, p=.547\right.$, partial $\eta^{2}=$ $.016]$, therefore the between-subjects factor was removed from the calculation.
} 
$\left[F(1,132)=3.029, p=.084\right.$, partial $\left.\eta^{2}=.022\right]$. Figure 2 shows the means for the four conditions. The proportion correctly solved was $.44(S D=.19)$ in the reinstated-zero practice condition, .38 in the reinstatedtwo repetition condition ( $S D=.2), .42$ in the new-zero repetition condition $(S D=.19)$, and .41 in the newtwo repetition condition $(S D=.18)$. Post-hoc paired comparisons of reinstated vs new context conditions were not significant in either the zero $[t(132)=.69, p=.49]$ or two retrieval practice $[t(132)=1.61, p=.11]$ condition.

Mean relatedness rating on a 1-10 scale between the context pictures and the red herrings, in which 1 meant extremely unrelated and 10 meant highly related, was $2.85(S D=1.6)$. A paired-samples t-test showed that participants during the second retrieval practice trial $(M=$ $.78, S D=.2)$ remembered the red herrings significantly better $[t(255)=37.37, p<.001, d=2.34]$ than during the first retrieval practice trial $(M=.46, S D=.18)$.

\section{Discussion}

Experiment 1 tested whether repetition and context reinstatement would modulate the strength of the fixation effect caused by presentation of red herrings. As predicted, with more retrieval practices of the red herrings, solution rates for the RAT items suffered. Two extra retrieval practice trials decreased solution rates by $4 \%$, an effect size of partial $\eta^{2}=.038$. Because Experiment 1 did not include an unprimed baseline condition, it is not clear how this effect compares with the overall fixation effect that may have occurred. Experiment 2 included an unprimed condition.

The main effect of context reinstatement was not significant. The methods that we used to observe contextdependent fixation have been shown to produce robust context-dependent memory effects (e.g., (Smith, 2013)). The context stimuli that we used were photographs of real places, making them "rich" enough to yield robust effects. There were only three paired-associates per context, a small contextual fan size. Our context encoding task ensured that items and contexts had been directly associated. These methods have yielded robust context-dependent memory effects for tests of free recall (Smith \& Manzano 2010, Smith et al., 2014), recognition (Shahabuddin \& Smith, 2016), and paired-associates recall (Smith \& Handy, 2014, 2016; Smith et al., 2014). Why, then, was the effect of context reinstatement not significant in Experiment 1?

One possible reason for our failure to detect context reinstatement-enhanced fixation, is, of course, that there may not be an effect of context reinstatement on experimentally-induced fixation effects. Alternatively, context reinstatement may truly influence fixation, but effect size may be much smaller than what we observed in Experiment 1 for the effect of repetition on fixation, and we may have had insufficient power to detect the effect. We suspected a different reason, however, based on the types of associations between contexts and red herrings, and between test words and red herring words. Whereas the associations between contexts and red herrings were purely arbitrary and episodic (e.g., a photo of an airfield and the red herring word $h u t$ ), the associations between test words and red herrings were semantic and meaningful (e.g., COTTAGE-hut). The stronger semantic associations, which existed even before participants enrolled in the experiment, were further strengthened in Experiment 1 by the paired-associates encoding task and the retrieval practice task. Thus, when the three test words on a RAT problem appeared, the presence of the strong word-word associates at test obscured the weaker effects of contextual stimuli, a

Fig. 2 Mean proportion of correct solutions for the RAT as a function of the four conditions in Experiment 1. The error bars represent $95 \%$ within-subjects CI
Context and Retrieval Practices

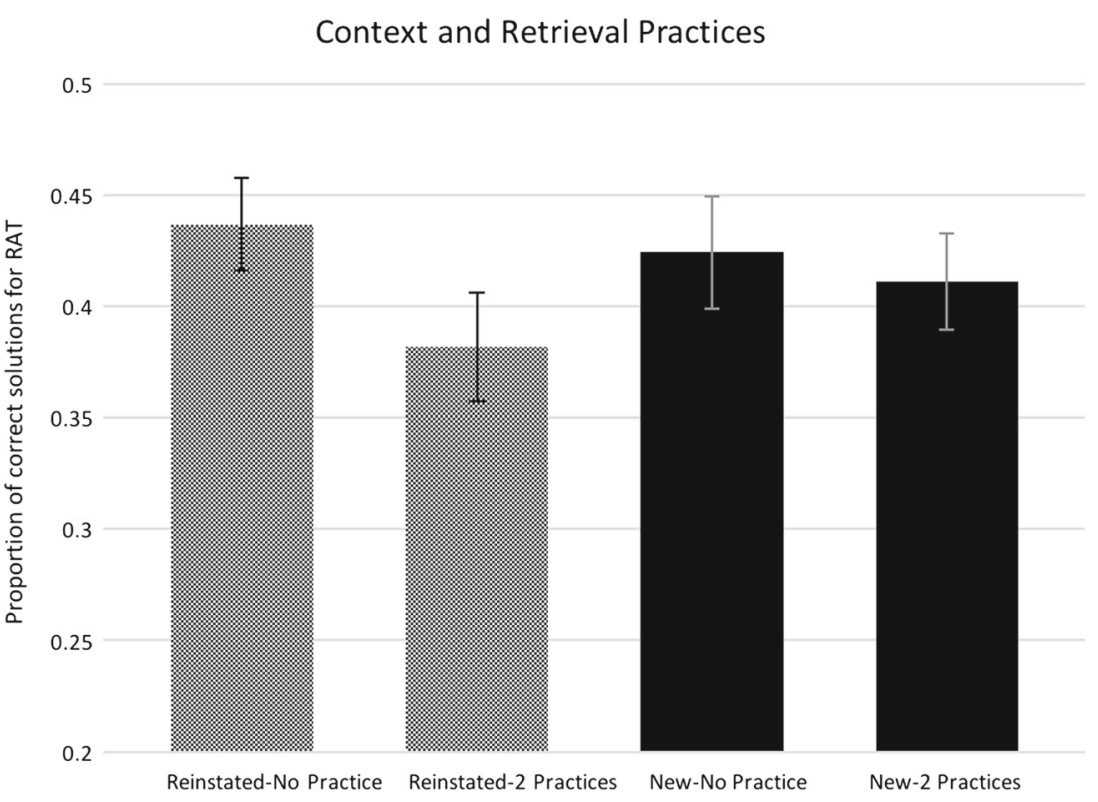


phenomenon known as an outshining effect (e.g., McDaniel et al., 1989; Smith \& Vela, 1986, 2001). An alternative to outshining might be that because of the strong association of the paired-associates, the contexts were never even encoded. The strongly associated red herrings overshadowed the arbitrary contexts at encoding (Pavlov, 1927, p.141). Although we did not test these two explanations per se, in Experiment 2 we removed the possibility of either outshining or overshadowing by having red herrings associated with contexts directly rather than pairing them with the RAT words as was done in Experiment 1.

Although the main effect of context and the context $\mathrm{X}$ repetition interaction were not significant in Experiment 1, the non-significant trend ( $p=.084$ ) suggests that greater effects of context manipulations may occur with more retrieval practice trials in a given context. This possibility is supported by the reasoning that more context-item practice strengthens not only the items themselves, but also the item-context associations, making memory of items more dependent upon the context. Therefore, in addition to mitigating potential outshining effects in Experiment 2, we also tried to maximize the likelihood of finding context-dependent fixation effects by using two contextitem retrieval practice trials for all items.

\section{Experiment 2}

In Experiment 1, triads of paired-associates, made from each RAT test word paired with its red herring (e.g., COTTAGE-hut, SWISS-chocolate, CAKE-icing), had been studied in association with photo contexts (e.g., a photo of an airfield). In Experiment 2, to avoid an outshining effect from powerful word cues, we instead paired the three red herrings for a subsequent problem (e.g., hut, chocolate, icing) with a photo context (e.g., an airfield). Participants were told that for each trial of a subsequent memory test, they would see a photograph, and they were to recall the three words that had been paired with the photo. Following two practice trials in which participants tried to recall the three words for each photo context (with post-trial corrective feedback), participants were given a test of RAT problems. A third of the RAT problems were displayed on the photo context that was paired with red herring words at encoding (primed-reinstated context), a third were shown superimposed over a new context (primednew context), and a third did not correspond to any red herrings shown in the experiment (unprimed items). The red herring retrieval hypothesis predicted that the unprimed RAT problems would have the highest solution rate, the primed-new context problems would have the next highest solution rate, and RAT problems in the primed-reinstated context condition would have the lowest solution rate.
Furthermore, following an analysis of solution rates of RAT problems from Experiment 1, 12 of the original set of 36 RAT items were excluded from Experiment 2 because their solution rates were too low to show significantly decreased performance in the fixation conditions. For Experiment 1, we selected items with high solution rates from the Bowden and Jung-Beeman (2003) norms (above $33 \%$ in the 15 seconds per problem condition), because we expected our fixation manipulations to decrease solution rates. In Experiment 1, twelve of the RAT items had mean solution rates below $10 \%$, a base rate too low to observe effects of fixation. Therefore we eliminated those 12 items from the stimulus materials used in Experiment 2. The remaining items in the Bowden and Jung-Beeman (2003) norm had baseline solution rates that were too low to observe fixation effects, therefore we used the reduced set of 24 items with high solution rates in Experiment 2.

\section{Method}

\section{Participants}

An a priori power analysis indicated a desired total sample size of 84 to detect a medium-sized (Cohen's $d=.5$ ) effect with an alpha level of .25 and power of .95. Participants were undergraduate psychology students volunteering as participants to fulfill part of a course requirement. They could enroll for time slots via a computerized database. The sessions were conducted with 3-15 participants at a time. There was a total of 89 participants in Experiment 2: 30 in CB-1, 31 in CB-2 and 28 in CB-3.

\section{Design and materials}

Test condition (primed-reinstated context, primed-new context and unprimed) was a within-subjects measure, and counterbalancing (CB-1, CB-2 and CB-3) was a betweensubjects measure. There were 24 items distributed in each test condition (see Appendix). In Experiment 2, the set of items (i.e., 8 RAT problems with their accompanying red herrings) in the primed-reinstated context condition in CB-1 were in the primed-new context condition in $\mathrm{CB}-2$, and the unprimed (baseline) condition in CB-3. The other two sets of 8 items were similarly counterbalanced among the three counterbalancing groups. The proportion of RAT problems solved was the dependent measure.

\section{Procedure}

The procedure used in Experiment 2 was the same as described for Experiment 1, with the following exceptions.

On the context encoding task in Experiment 2, participants were instructed to memorize each set of 3 words 


\section{Context encoding}

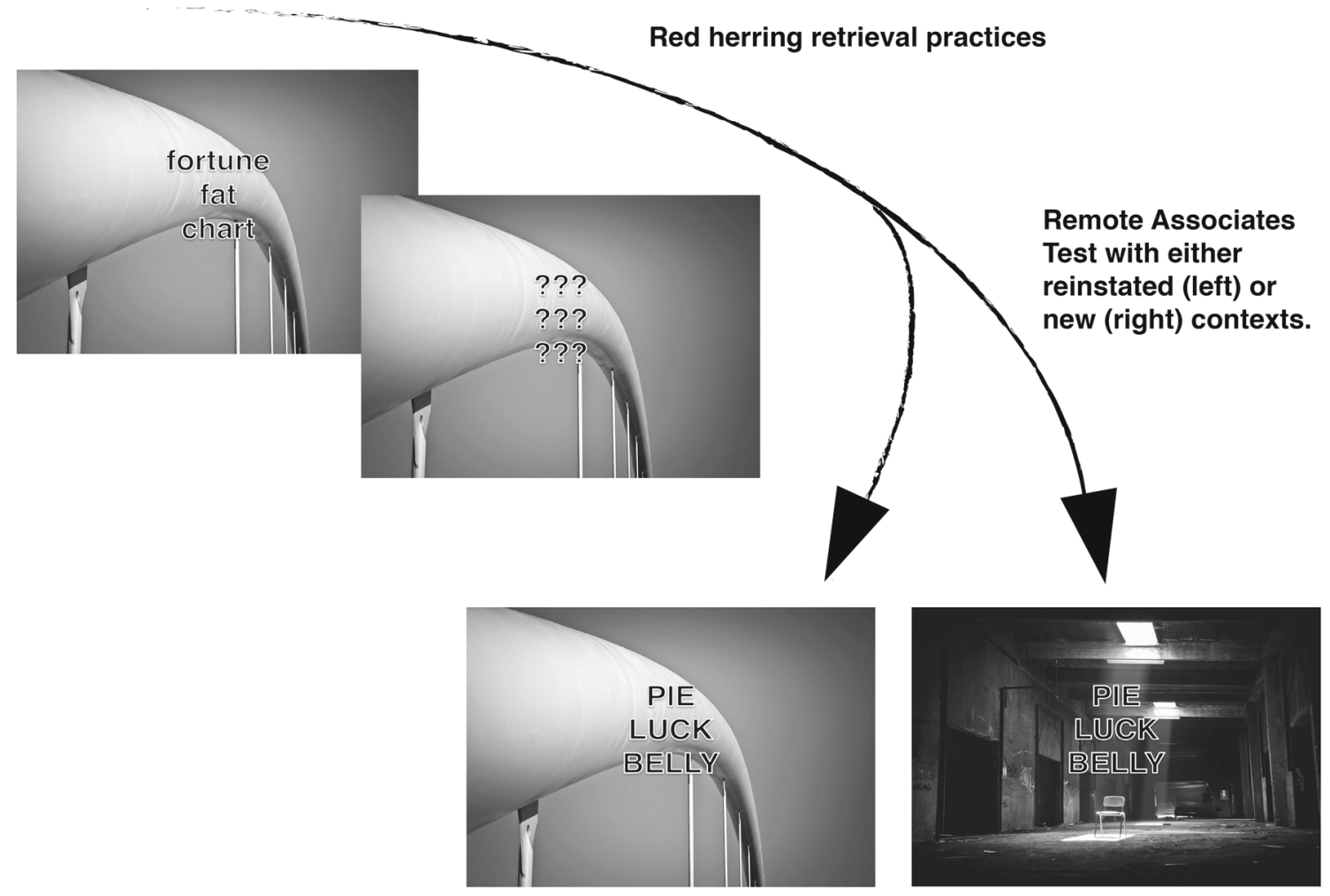

Fig. 3 Experiment 2 method

appearing together on the screen (i.e., the 3 red herrings for a RAT problem) in association with the context photos.

For each trial of the retrieval practice task in Experiment 2, participants were shown one of the encoded photo contexts as a cue, and they tried to recall the three words that they had encoded on the previous task. Participants wrote these responses on paper, with 10 -sec per retrieval practice trial. After 10 -sec, the correct responses were shown on the screen, participants either circled the responses that they got correct on that trial, or they wrote down the responses they could not remember. This self-correction procedure took another 10sec per trial. All 16 of the encoded items were tested in this way twice. The method of Experiment 2 is shown on Fig. 3.

\section{Results}

A repeated measures one-way ANOVA was computed, to test the effects of test position (unprimed, primed-reinstated context, primed-new context), using the proportion of RAT problems solved as a dependent variable. ${ }^{2}$ There

\footnotetext{
${ }^{2}$ The inclusion of counterbalancing in a mixed ANOVA as a betweensubjects measure showed no significant differences between the three counterbalancing groups $\left[F(2,86)=1.025, p=.363\right.$, partial $\eta^{2}=$ $.023]$, therefore the between-subjects factor was removed from the calculation.
}

was a significant difference among the three conditions $\left[F(2,176)=8.127, p<.001\right.$, partial $\left.\eta^{2}=.085\right]$ Post-hoc pairwise comparisons with a Bonferroni correction revealed, that compared to the unprimed condition $(M=$ $.539, S D=.195)$, solutions for the RAT problems were significantly worse when the encoding contexts of red herrings at the test phase were reinstated $(M=.456$, $S D=.205)[t(88)=4.011, p<.001, d=.425]$. We also found that compared to the new context condition $(M=.506, S D=.205)$ there was a significant decline in correct solutions if the encoding contexts of red herrings was reinstated at test $[t(88)=2.585, p=.034, d=$ .274]. The difference between the unprimed and primednew context conditions was not significant $[t(88)=1.517$, $p=.399, d=.161]$ (Fig. 4).

A paired-samples t-test showed that participants during the second retrieval practice trial $(M=.58, S D=.24)$ remembered the red herrings significantly better $[t(169)=$ 21.64, $p<.001, d=1.66]$ than during the first retrieval practice trial $(M=.22, S D=.15)$.

\section{Discussion}

In contrast with the results of Experiment 1, there was a clear context-dependent fixation effect observed in Experiment 2. 
Fig. 4 Mean proportion of correct solutions for the RAT as a function of the three context conditions in Experiment 2. The error bars represent $95 \%$ within-subjects CI
Reinstated vs New Contexts with Unprimed condition

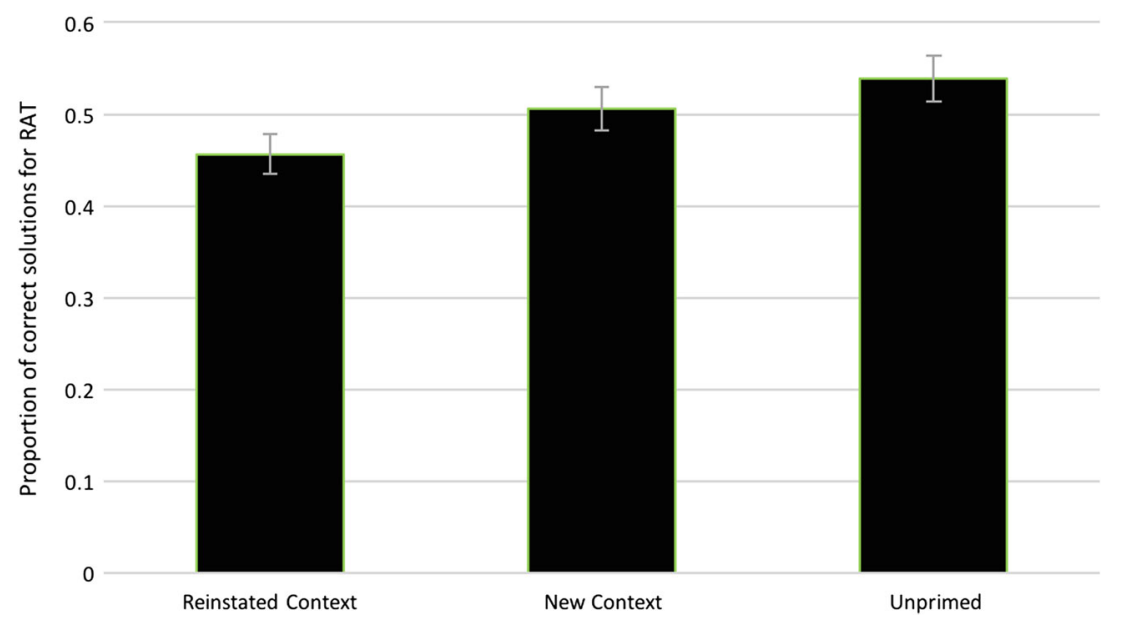

The RAT problems tested with the encoding contexts of corresponding red herrings (i.e., the primed-reinstated context items), relative to RAT problems tested with new contexts (i.e., the primed-new context items), were solved at a significantly lower rate. The primary difference between the procedures used in Experiments 1 and 2 was that in Experiment 1, both RAT test words and photo contexts were practiced in association with red herrings, whereas in Experiment 2, only photo contexts were practiced with red herrings. In Experiment 1, the strong semantic associations between RAT test words and red herring words were made even stronger because they were practiced together. The presence of those strong verbal cues at test, regardless of the test context, caused an outshining effect (e.g., Smith, 1988) in Experiment 1. Because the associations between test words and red herrings were practiced in Experiment 1, but not Experiment 2, the verbal cues were much stronger in Experiment 1 than in Experiment 2. As a result, the cuing effects of photo contexts were not outshone by the stronger cuing effects of RAT test words in Experiment 2, as they were in Experiment 1. In addition, all items received one presentation trial and two retrieval practice trials before the critical RAT problems, based upon the suggestion in the results of Experiment 1 that multiple context-item repetitions may strengthen the context-red herring associations, making them more context-dependent.

In Experiment 2, we included a baseline control condition (i.e., the unprimed condition) in which there were no corresponding red herrings that had been encoded and practiced before the RAT was given. The percentage of RAT problems solved in the primed-reinstated context condition, relative to the unprimed condition, was $8 \%$ lower, a fixation effect size ( $d=.43$ ) in the medium range (Cohen, 1977). In contrast, the $3 \%$ fixation effect in the primed-new context condition did not rise to significance, with a very small effect size $(d=.16)$. In addition to showing that a test manipulation, context reinstatement, affects the magnitude effect, the present findings suggest that trying to solve creative problems in new contexts may be a way to relieve fixation.

\section{General discussion}

Our results are consistent with the red herring retrieval hypothesis, the notion that red herrings retrieved from memory cause fixation effects, and furthermore, that factors that improve memory of red herrings also increase fixation effects. When the test words of RAT problems (e.g., COTTAGE - SWISS - CAKE), acting as stimulus members of paired associates, were practiced with red herrings (e.g., hut, chocolate, icing), that is, words conceptually related to test words but unrelated to solutions (e.g., cheese), performance on the RAT problems decreased, a fixation or blocking effect. Furthermore, when test word-red herring pairs were practiced more (Experiment 1), and when the encoding contexts of red herrings were reinstated (Experiment 2) the observed fixation effect was greater. The degree of practice or repetition should affect an item's storage strength (Bjork \& Bjork, 1992), or that item's potential for being remembered, whereas providing context cues should affect the item's retrieval strength, or the amount of storage potential that can be utilized. Our experiments show that manipulations of both the storage and retrieval strength of red herrings can affect the degree of fixation that is observed.

A not completely unexpected result was the lack of a context-dependent fixation effect in Experiment 1. Previous research has shown that cued recall, using paired associates, is susceptible to the types of context manipulations that we used in the present experiments (e.g., Smith et al., 2014; Smith \& Handy, 2014, 2016). Smith et al. (2014), for example, observed context-dependent cued recall using Swahili-English word pairs, Smith and Handy (2014) found the effect using face-name pairs, and Smith and Handy (2016) 
found it using Tagalog-English word pairs. The word pairs used in Experiment 1 of the present study, however, differed from previously reported methods because the two words of our pairs were in the same language, semantically related to each other (e.g., COTTAGE-hut, SWISS-chocolate, CAKEicing). The presence of strong semantic cues at test has been shown to weaken or eliminate episodic context reinstatement effects, consistent with the outshining hypothesis (Smith \& Vela, 2001). Consistent with this reasoning, a clear effect of context reinstatement on fixation was seen in Experiment 2 when red herrings were associated with unrelated context cues, rather than with semantically related RAT word cues, at encoding. Another reason that context effects were significant in Experiment 2, but not in Experiment 1, might have been that each of the red herrings in Experiment 2 were presented and practiced twice, all in one single context, and the repeated association of contexts with red herrings might have made them more context-dependent, a notion consistent with the non-significant interaction of context $\mathrm{X}$ repetition observed in Experiment 1. Although these explanations are logical, they are also speculative; the present experiments do not provide a critical test of them.

Our experiments broaden the linkage of research on creative thinking with memory research (e.g., Storm \& Koppel, 2012; Koppel \& Storm, 2014; Smith \& Blankenship, 1989, 1991; Storm \& Angello, 2010; Storm, Angello, \& Bjork, 2011; Angello, Storm, \& Smith, 2015). The sensitivity of RAT problem solving, a test often used to assess creative ability (e.g., Benedek \& Neubauer, 2013), to manipulations of the memorability of red herrings in the present two experiments shows that this test of creative problem solving has acted as an indirect measure of memory (Richardson-Klavehn \& Bjork, 1988). In contrast to direct memory measures, indirect measures are those that do not refer participants to the items being remembered, but that are nonetheless influenced by memory of those items. Performance on tests of word fragment completion and anagrams, for example, reflect the manipulated memorability of solutions to those simple types of problems (e.g., Graf \& Schacter, 1985; Kelley, Jacoby, \& Hollingshead, 1989), as well as the manipulated memorability of red herrings (e.g., Landau \& Leynes, 2004; Smith \& Tindell, 1997). It is not clear whether the fixation effects observed in the present experiments were caused by automatic retrieval or deliberate recollection of red herrings. It is clear, however, that most participants knew that red herrings were not correct solutions; red herrings were given as intrusions on only $1.85 \%$ of all RAT problems in Experiment 1, and on only $0.79 \%$ of the problems in Experiment 2. The degree to which the present findings are due to conscious recollection of red herrings, versus how much is due to unconscious, automatic retrieval, should be a focus of future research.

Implicit memory blocking effects have been mitigated by weakening the retrieval strength of red herrings (or blockers) via memory inhibition procedures, including directed forgetting (Koppel \& Storm, 2012) and suppression induced forgetting (Angello et al., 2015) of red herrings (e.g., ANALOGY) that were designed to block word fragment completion (e.g., A_L_GY). an indirect test of memory (see Smith \& Tindell, 1997). Mitigating fixation in creative problem solving might be accomplished the same way, that is, by inhibiting the associations between problem stimuli and red herrings. This possibility is consistent with findings that show significant correlations between an individual's RAT problem solving performance and the magnitude of that individual's retrieval-induced forgetting effect; perhaps individuals who are good at inhibiting memories are also good at inhibiting inappropriate responses that may come to mind (and cause fixation) while trying to solve RAT problems. The present findings, that repetitions and context reinstatement of red herrings increased fixation in creative (RAT) problem solving, are consistent with the idea that better retrieval of red herrings increases their blocking (i.e., fixation) effectiveness.

Our experimental manipulations of participants' memory of red herrings, which moderated blocks in creative problem solving, is consistent with a considerable body of research on creative cognition that has linked fixation and constraints that limit creative thinking with the inappropriate use of prior knowledge. The fixating effects of implicit assumptions has been explored and discussed at length by Gestalt psychologists, an acknowledgment that prior knowledge can cause fixation effects such as mental set (or Einstellung), (Luchins \& Luchins, 1959; Duncker, 1945; Koffka, 1935; Köhler, 1925; Wertheimer, 1945) or functional fixity (e.g., Adamson \& Taylor, 1954; Maier, 1931). In a similar vein, in constraint relaxation theory (e.g., Knoblich et al., 1999) knowledge about what does and does not serve as a satisfactory solution to a creative problem is described in terms of constraints, with such constraints having been learned and remembered from experiences with similar problems in one's past. Likewise, the theory of structured imagination (Ward, 1994), which states that creative imagination is based on one's conceptual knowledge, describes prematurely constrained idea development resulting from taking the "path of least resistance" after beginning with an overly constrained concept. And, using RAT problems whose incorrect solutions were baseball terms Wiley (1998) showed that prior knowledge about baseball could impede creative problem solving. What the present study adds to this body of research is the use of experimental paradigms that manipulate the memory of red herrings, or inappropriate solutions.

The red herring retrieval theory, which states that creative problem solving performance depends on the retrieval strength of red herrings, is closely related to Smith and Blankenship's forgetting fixation theory of incubation effects. This theory of incubation states that a decrease in memory of 
fixating ideas is the cause of incubation effects, defined as resolution of initial blocks (or fixation) as a function of forgetting red herrings (blockers) during the incubation interval. Smith and Blankenship (1989), for example, showed that longer incubation intervals caused both poorer memory of blockers and better resolution of initially unsolved rebus problems, consistent with the forgetting fixation theory of incubation effects. Theories that link the passage of time with changes in one's mental context, that is, contextual fluctuation theories (Estes, 1955; Bower, 1972; Glenberg, 1979; Howard \& Kahana, 2002; Mensink \& Raaijmakers, 1989), are consistent with the notion that contextual fluctuation, after initial fixation on a problem, rather than the passage of time per se, may be what mitigates fixation during an incubation period. The findings of context-dependent fixation in Experiment 2 of the present study indicate that initial fixation might be mitigated by a change of contexts, regardless of the passage of time. Such an explanation of "incubation" effects suggests a method of enhancing incubation effects may be to present initially unsolved problems in new contexts.

In conclusion, we have shown that creative problem solving is impeded by manipulations of factors that improve memory of red herrings for those problems. In Experiment 1, repeated practice of associations between problems and red herrings caused greater fixation. In Experiment 2, practice of associations between contexts and red herrings caused greater fixation for problems tested in those practiced contexts. The fact that retrieval of fixating ideas can be triggered by problems or by contexts shows that there can be multiple sources of fixation in creative problem solving, and indicates that efforts to reduce or overcome fixation might focus on memory cues provided by problems and/or by problem solving contexts.

\section{Appendix: Remote associates test items and their red herrings}

\begin{tabular}{|c|c|c|c|c|c|c|c|c|c|c|c|}
\hline & Solution & RAT problem & Red herrings & & & & & & & & \\
\hline \multirow[t]{3}{*}{1} & Cheese & COTTAGE & Hut & $13^{*}$ & Fire & CRACKER & Nut & $25^{*}$ & Pot & PIE & Chart \\
\hline & & SWISS & Chocolate & & & FLY & Kite & & & LUCK & Fortune \\
\hline & & CAKE & Icing & & & FIGHTER & Soldier & & & BELLY & Fat \\
\hline \multirow[t]{3}{*}{$2 *$} & Ice & CREAM & Butter & $14 *$ & Pin & SAFETY & Belt & $26^{*}$ & Soap & OPERA & Soprano \\
\hline & & SKATE & Board & & & CUSHION & Couch & & & HAND & Fingers \\
\hline & & WATER & Hose & & & POINT & Score & & & DISH & Satellite \\
\hline \multirow[t]{3}{*}{$3^{*}$} & Sore & LOSER & Winner & $15^{*}$ & Sugar & CANE & Crutch & 27 & Space & CADET & Corps \\
\hline & & THROAT & Swallow & & & DADDY & Mommy & & & CAPSULE & Pill \\
\hline & & SPOT & Notice & & & PLUM & Purple & & & SHIP & Cruise \\
\hline \multirow[t]{3}{*}{$4 *$} & Boat & SHOW & Movie & 16 & Blind & DATE & Time & 28 & Sick & HOME & Plate \\
\hline & & LIFE & Death & & & ALLEY & Path & & & SEA & Turtle \\
\hline & & ROW & Dispute & & & FOLD & Center & & & BED & Sheet \\
\hline \multirow[t]{3}{*}{$5 *$} & Watch & NIGHT & Crawler & 17 & Gold & FISH & Hook & $29 *$ & Coat & FUR & Otter \\
\hline & & WRIST & Flick & & & MINE & Yours & & & RACK & Torture \\
\hline & & STOP & Signal & & & RUSH & Hurry & & & TAIL & Spin \\
\hline \multirow[t]{3}{*}{$6^{*}$} & Camp & BOOT & Cowboy & $18^{*}$ & Party & POLITICAL & Office & 30 & Blood & HOUND & Canine \\
\hline & & SUMMER & Season & & & SURPRISE & Attack & & & PRESSURE & Cooker \\
\hline & & GROUND & Coffee & & & LINE & Up & & & SHOT & Gun \\
\hline \multirow[t]{3}{*}{$7 *$} & Chair & ROCKING & Rolling & $19^{*}$ & Common & SENSE & Vision & $31 *$ & Hole & FOX & Hunt \\
\hline & & WHEEL & Axle & & & COURTESY & Manners & & & MAN & Primate \\
\hline & & HIGH & Priest & & & PLACE & Mat & & & PEEP & Chicken \\
\hline \multirow[t]{3}{*}{8} & Honey & DEW & Morning & $20 *$ & Book & WORM & Earth & 32 & Family & NUCLEAR & Bomb \\
\hline & & COMB & Hair & & & SHELF & Storage & & & FEUD & Argument \\
\hline & & BEE & Spelling & & & END & Deadline & & & ALBUM & Music \\
\hline \multirow[t]{3}{*}{9} & Soda & FOUNTAIN & Youth & 21 & Copy & RIGHT & Left & $33 *$ & Bag & SLEEPING & Pillow \\
\hline & & BAKING & Oven & & & CAT & Mouse & & & BEAN & Kidney \\
\hline & & POP & Star & & & CARBON & Oxygen & & & TRASH & Garbage \\
\hline \multirow[t]{3}{*}{$10 *$} & Forest & PRESERVE & Jelly & $22 *$ & Girl & FLOWER & Daisy & $34 *$ & Fast & FOOD & Hungry \\
\hline & & RANGER & Texas & & & FRIEND & Enemy & & & FORWARD & Backward \\
\hline & & TROPICAL & Heat & & & SCOUT & Ahead & & & BREAK & Shatter \\
\hline \multirow[t]{3}{*}{$11 *$} & Band & AID & Cool & $23^{*}$ & Bank & RIVER & Canoe & $35^{*}$ & Moon & SHINE & Armor \\
\hline & & RUBBER & Tire & & & NOTE & Worthy & & & BEAM & Laser \\
\hline & & WAGON & Station & & & ACCOUNT & Receipt & & & STRUCK & Hit \\
\hline \multirow[t]{3}{*}{12} & Snow & FLAKE & Weirdo & $24 *$ & Blue & PRINT & Press & 36 & Pit & PEACH & Fuzz \\
\hline & & MOBILE & Phone & & & BERRY & Bush & & & ARM & Mechanical \\
\hline & & CONE & Traffic & & & BIRD & Nest & & & TAR & Heel \\
\hline
\end{tabular}




\section{References}

Adamson, R. E., \& Taylor, D. W. (1954). Functional fixedness as related to elapsed time and to set. Journal of Experimental Psychology, 47(2), 122-126. ISSN: 0022-1015. https://doi.org/10.1037/h0057 297

Angello, G., Storm, B. C., \& Smith, S. M. (2015). Overcoming fixation with repeated memory suppression. Memory, 23(3), 381-389. ISSN: 1464-0686 (Electronic) 0965-8211 (Linking). https://doi.org/ 10.1080/09658211.2014.889167. https://www.ncbi.nlm.nih.gov/ pubmed/24575886

Benedek, M., \& Neubauer, A. C. (2013). Revisiting Mednick's model on creativity-related differences in associative hierarchies. Evidence for a common path to uncommon thought. Journal of Creative Behavior, 47(4), 273-289. ISSN:0022-0175 (Print) 00220175 (Linking). https://doi.org/10.1002/jocb.35. https://www.ncbi. nlm.nih.gov/pubmed/24532853

Bjork, R. A., \& Bjork, E. L. (1992). A new theory of disuse and an old theory of stimulus fluctuation. In Healy, A. F., Kosslyn, S. M., \& Shiffrin, R. M. (Eds.) Essays in honor of William K. Estes, Vol. 1: From learning theory to connectionist theory; Vol. 2: From learning processes to cognitive processes. ISBN: 0-8058-10978 (Hardcover) 0-8058-0759-4 (Hardcover). Hillsdale: Lawrence Erlbaum Associates Inc.

Bowden, E. M., \& Jung-Beeman, M. (2003). Normative data for 144 compound remote associate problems. Behavior Research Methods Instruments \& Computers, 35(4), 634-639. ISSN: 0743-3808. $<$ GotoISI > ://WOS:000187916000017. https://doi.org/10.3758/bf 03195543

Bower, G. H. (1972). Perceptual groups as coding units in immediate memory. Psychonomic Science, 27(4), 217-219. ISSN: 00333131. https://doi.org/10.3758/bf03328942

Chrysikou, E. G., \& Weisberg, R. W. (2005). Following the wrong footsteps: fixation effects of pictorial examples in a design problem-solving task. Journal of Experimental Psychology: Learning Memory and Cognition, 31(5), 1134-1148. ISSN: 19391285. < GotoISI > ://WOS:000233055100021. https://doi.org/10.1 037/0278-7393.31.5.1134

Cohen, J. (1977). Statistical power analysis for the behavioral sciences, Rev. ed. Hillsdale: Lawrence Erlbaum Associates, Inc. ISBN: 0-12-179060-6. http://p2048-lib-ezproxy.tamu.edu.ezproxy. library.tamu.edu/login? http://search.ebscohost.com/login.aspx?di rect=true $\& d b=$ psyh $\& A N=1987-98267-000 \&$ site=ehost-live

Duncker, K. (1945). On problem-solving. 58, i-113. ISSN: 0096-9753. https://doi.org/10.1037/h0093599.

Estes, W. K. (1955). Statistical theory of spontaneous recovery and regression. Psychological Review, 62(3), 145-154. ISSN: 19391471 0033-295X. https://doi.org/10.1037/h0048509

Finke, R. A., Ward, T. B., \& Smith, S. M. (1992). Creative cognition: Theory, research, and applications, (p. 239). Cambridge: The MIT Press. ISBN: 0-262-06150-3 (Hardcover).

Glenberg, A. M. (1979). Component-levels theory of the effects of spacing of repetitions on recall and recognition. Memory \& Cognition, 7(2), 95-112. ISSN: 0090-502X 1532-5946. https://doi.org/ 10.3758/bf03197590

Graf, P., \& Schacter, D. L. (1985). Implicit and explicit memory for new associations in normal and amnesic subjects. Journal of Experimental Psychology: Learning, Memory, and Cognition, 11(3), 501-518. ISSN: 1939-1285 0278-7393. https://doi.org/10. 1037/0278-7393.11.3.501

Howard, M. W., \& Kahana, M. J. (2002). A distributed representation of temporal context. Journal of Mathematical Psychology, 46(3), 269-299. ISSN: 0022-2496. <GotoISI>://WOS:000176 738900002. https://doi.org/10.1006/jmps.2001.1388
Jacoby, L. L. (1996). Dissociating automatic and consciously controlled effects of study/test compatibility. Journal of Memory and Language, 35(1), 32-52. ISSN: 0749-596X. https://doi.org/10. 1006/jmla.1996.0002

Jacoby, L. L. (1998). Invariance in automatic influences of memory: Toward a user's guide for the process-dissociation procedure. Journal of Experimental Psychology: Learning, Memory and Cognition, 24(1), 3-26. ISSN: 0278-7393. https://doi.org/10.1037// 0278-7393.24.1.3. https://www.ncbi.nlm.nih.gov/pubmed/9438951

Jacoby, L. L., \& Dallas, M. (1981). On the relationship between autobiographical memory and perceptual learning. Journal of Experimental Psychology: General, 110(3), 306-340. ISSN: 0096-3445. https://doi.org/10.1037//0096-3445.110.3.306

Jacoby, L. L., Toth, J. P., \& Yonelinas, A. P. (1993). Separating conscious and unconscious influences of memory: Measuring recollection. Journal of Experimental Psychology: General, 122(2), 139-154. ISSN: 0096-3445. <GotoISI>://WOS:A199 3LD27100001. https://doi.org/10.1037//0096-3445.122.2.139

Jansson, D. G., \& Smith, S. M. (1991). Design fixation. Design Studies, 12(1), 3-11. ISSN: 0142-694X. https://doi.org/10.1016/0142$694 \mathrm{x}(91) 90003-\mathrm{f}$

Kelley, C. M., Jacoby, L. L., \& Hollingshead, A. (1989). Direct versus indirect tests of memory for source: Judgments of modality. Journal of Experimental Psychology: Learning, Memory, and Cognition, 15(6), 1101-1108. ISSN: 0278-7393. https://doi.org/10.1037// 0278-7393.15.6.1101

Kirsner, K., Milech, D., \& Standen, P. (1983). Common and modalityspecific processes in the mental lexicon. Memory \& Cognition, 11(6), 621-630. ISSN: 0090-502X. https://doi.org/10.3758/bf031 98287

Knoblich, G. et al. (1999). Constraint relaxation and chunk decomposition in insight problem solving. Journal of Experimental Psychology: Learning, Memory, and Cognition, 25(6), 1534-1555. ISSN: 0278-7393. https://doi.org/10.1037//0278-7393.25.6.1534

Koffka, K. (1935). Principles of gestalt psychology. London: Lund Humphries. https://doi.org/10.2307/2180493

Köhler, W. (1925). The mentality of apes. Trans. by Ella Winter. Oxford: Harcourt, Brace https://doi.org/10.1038/116351a0.

Kohn, N. W., \& Smith, S. M. (2009). Partly versus completely out of your mind: Effects of incubation and distraction on resolving fixation. Journal of Creative Behavior, 43(2), 102-118. ISSN 0022-0175. https://doi.org/10.1002/j.2162-6057.2009.tb01309.x

Kohn, N. W., \& Smith, S. M. (2011). Collaborative fixation: Effects of others' ideas on brainstorming. Applied Cognitive Psychology, 25(3), 359-371. ISSN: 08884080. https://doi.org/10.1002/acp.1699

Koppel, R. H., \& Storm, B. C. (2012). Unblocking memory through directed forgetting. Journal of Cognitive Psychology, 24(8), 901907. ISSN: 2044-5911 2044-592X. https://doi.org/10.1080/20445 911.2012.716822

Koppel, R. H., \& Storm, B. C. (2014). Escaping mental fixation: Incubation and inhibition in creative problem solving. Memory, 22(4), 340-348. ISSN: 1464-0686 (Electronic) 09658211 (Linking). https://doi.org/10.1080/09658211.2013.789914. https://www.ncbi.nlm.nih.gov/pubmed/23607286

Landau, J. D., \& Leynes, P. A. (2004). Manipulations that disrupt generative processes decrease conformity to examples: Evidence from two paradigms. Memory, 12(1), 90-103. ISSN: 09658211. https://doi.org/10.1080/09658210244000388. https://www. ncbi.nlm.nih.gov/pubmed/15098623

Landau, J. D., \& Leynes, P. A. (2006). Do explicit memory manipulations affect the memory blocking effect? The American Journal of Psychology, 119(3), 463. ISSN: 0002-9556. https://doi.org/10.2307/20445353

Leynes, P. A., Brown, J., \& Landau, J. D. (2011). Objective and subjective measures indicate that orthographically similar words produce 
a blocking experience. Memory, 19(1), 17-35. ISSN: 0965-8211. https://doi.org/10.1080/09658211.2010.531025. https://www.ncbi. nlm.nih.gov/pubmed/21240746

Logan, J. M., \& Balota, D. A. (2003). Conscious and unconscious lexical retrieval blocking in younger and older adults. Psychology and Aging, 18, 537-550. ISSN: 1939-1498. https://doi.org/10.1037/ 0882-7974.18.3.537

Luchins, A. S., \& Luchins, E. H. (1959). Rigidity of behavior, a variational approach to the effect of Einstellung. University of Oregon monographs, studies in psychology, (p. xxv, 623). Eugene: University of Oregon Books. https://doi.org/10.2307/1419434.

Lustig, C., \& Hasher, L. (2001). Implicit memory is vulnerable to proactive interference. Psychological Science, 12(5), 408412. ISSN: 0956-7976. https://doi.org/10.1111/1467-9280.00375. https://www.ncbi.nlm.nih.gov/pubmed/11554675

Maier, N. R. F. (1931). Reasoning in humans. II. The solution of a problem and its appearance in consciousness. Journal of Comparative Psychology, 12(2), 181-194. ISSN: 0093-4127. https://doi. org/10.1037/h0071361

McDaniel, M. A. et al. (1989). Modulation of environmental reinstatement effects through encoding strategies. The American Journal of Psychology, 102(4), 523. ISSN: 0002-9556. https://doi.org/10.230 7/1423306

Mednick, S. (1962). The associative basis of the creative process. Psychological Review, 69, 220-232. ISSN: 0033-295X. https://doi.org/10.1037/h0048850. https://www.ncbi.nlm.nih.gov/ pubmed/14472013

Mensink, G.-J. M., \& Raaijmakers, J. G. W. (1989). A model for contextual fluctuation. Journal of Mathematical Psychology, 33(2), 172-186. ISSN: 00222496. https://doi.org/10.1016/0022-2496(89) 90029-1

Müller, G. E., \& Pilzecker, A. (1900). Experimentelle Beiträge zur Lehre vom Gedächtnis. German. Zeitschrift für Psychologie. Ergänzungsband, 1, 1-300.

Mulligan, N. W. (2011). Conceptual implicit memory and environmental context. Consciousness and Cognition, 20(3), 737-744. ISSN: 10538100. https://doi.org/10.1016/j.concog.2010.11.008

Neill, W. T., \& Valdes, L. A. (1992). Persistence of negative priming: Steady state or decay? Journal of Experimental Psychology: Learning, Memory, and Cognition, 18(3), 565-576. ISSN: 02787393. https://doi.org/10.1037//0278-7393.18.3.565

Parker, A., Gellatly, A., \& Waterman, M. (1999). The effect of environmental context manipulation on memory: Dissociation between perceptual and conceptual implicit tests. European Journal of Cognitive Psychology, 11(4), 555-570. ISSN: 0954-1446. https:// doi.org/10.1080/095414499382291

Pavlov, I. (1927). Conditioned reflexes. New York: Oxford University Press.

Purcell, A. T. et al. (1993). Fixation effects: Do they exist in design problem solving? Environment and Planning B: Planning and Design, 20(20), 333-345. ISSN: 0265-8135. https://doi.org/10. $1068 / \mathrm{b} 200333$

Richardson-Klavehn, A., \& Bjork, R. A. (1988). Measures of memory. Annual Review of Psychology, 39(1), 475-543. ISSN: 0066-4308 1545-2085. https://doi.org/10.1146/annurev.ps.39.020188.002355

Scarborough, D. L., Gerard, L., \& Cortese, C. (1979). Accessing lexical memory: The transfer of word repetition effects across task and modality. Memory \& Cognition, 7(1), 3-12. ISSN: 0090 -502X. https://doi.org/10.3758/bf03196928

Shahabuddin, S. S., \& Smith, S. M. (2016). Asymmetric reinstatement effects in recognition. Journal of General Psychology, 143(4), 267-280. ISSN: 1940-0888 (Electronic) 0022-1309 (Linking). https://doi.org/10.1080/00221309.2016.1214100. https: //www.ncbi.nlm.nih.gov/pubmed/27649359

Sio, U. N., Kotovsky, K., \& Cagan, J. (2015). Fixation or inspiration? A meta-analytic review of the role of examples on design processes. Design Studies, 39, 70-99. ISSN: 0142-694X. https://doi.org/10.1016/j.destud.2015.04.004

Smith, S. M. (1979). Remembering in and out of context. Journal of Experimental Psychology, 5(5), 460-471. ISSN: 0096-1515. https://doi.org/10.1037/0278-7393.5.5.460

Smith, S. M. (1988). Environmental context-dependent memory. In Thomson, D. M., \& Davies, G. M. (Eds.) Chap. 2, (pp. 13-34). New York: Wiley.

Smith, S. M. (1995a). Creative cognition: Demystifying creativity. In Hedley, C. N., Antonacci, P., \& Rabinowitz, M. (Eds.) The mind at work in the classroom: Literacy and thinking. Chap. 3, (pp. 31-46). Hillsdale: Erlbaum.

Smith, S. M. (1995b). Fixation, incubation, and insight in memory, problem solving, and creativity. In Smith, S. M., Ward, T. B., \& Finke, R. A. (Eds.) The creative cognition approach. ISBN: 0-262-19354-X, (pp. 135-156). Cambridge: The MIT Press.

Smith, S. M. (2003). The constraining effects of initial ideas. In Paulus, P. B., \& Nijstad, B. A. (Eds.) Group creativity: Innovation through collaboration. ISBN: 0-19-514730-8 (Hardcover). (pp. 1531). New York: Oxford University Press. https://doi.org/10.1093/ acprof:oso/9780195147308.003.0002.

Smith, S. M. (2013). Effects of environmental context on human memory. In Perfect, T. J., \& Lindsay, D. S. (Eds.) The SAGE handbook of applied memory, SAGE, 2013. ISBN: 1446296989, (pp. 162-182).

Smith, S. M., \& Blankenship, S. E. (1989). Incubation effects. Bulletin of Psychonomic Society, 27(4), 311-314. ISSN: 00905054. https://doi.org/10.3758/bf03334612

Smith, S. M., \& Blankenship, S. E. (1991). Incubation and the persistence of fixation in problem solving. The American Journal of Psychology, 104(1), 61-87. ISSN: 0002-9556. https://doi.org/10.230 7/1422851. https://www.ncbi.nlm.nih.gov/pubmed/2058758

Smith, S. M., \& Handy, J. D. (2014). Effects of varied and constant environmental contexts on acquisition and retention. Journal of Experimental Psychology: Learning, Memory, and Cognition, 40(6), 1582-1593. ISSN: 1939-1285. https://doi.org/10.1037/xlm 0000019

Smith, S. M., \& Handy, J. D. (2016). The crutch of contextdependency: Effects of contextual support and constancy on acquisition and retention. Memory, 24(8), 1134-1141. ISSN: 09658211. https://doi.org/10.1080/09658211.2015.1071852

Smith, S. M., Heath, F. R., \& Vela, E. (1990). Environmental contextdependent homophone spelling. The American Journal of Psychology, 103(2), 229-242. ISSN: 0002-9556. https://doi.org/10.2307/ 1423144

Smith, S. M., \& Manzano, I. (2010). Video context-dependent recall. Behavior Research Methods, 42(1), 292-301. ISSN: 1554351X. https://doi.org/10.3758/brm.42.1.292. https://www.ncbi.nl m.nih.gov/pubmed/20160308

Smith, S. M., \& Tindell, D. R. (1997). Memory blocks in word fragment completion caused by involuntary retrieval of orthographically related primes. Journal of Experimental Psychology: Learning, Memory, and Cognition, 23(2), 355-370. ISSN: 0278-7393. https://doi.org/10.1037//0278-7393.23.2.355

Smith, S. M., \& Vela, E. (1986). Outshining - the relative effectiveness of cues. Bulletin of the Psychonomic Society, 24(5), 350.

Smith, S. M., \& Vela, E. (2001). Environmental context-dependent memory: A review and meta-analysis. Psychonomic Bulletin \& Review, 8(2), 203-220. ISSN: 1069-9384. https://doi.org/10.3758/ bf03196157

Smith, S. M., Ward, T. B., \& Schumacher, J. S. (1993). Constraining effects of examples in a creative generation task. Memory \& Cognition, 21(6), 837-845. ISSN: 0090-502X. https://doi.org/10.375 8/bf03202751 
Smith, S. M. et al. (2014). Effects of similarity on environmental context cueing. Memory, 22(5), 493-508. ISSN: 1464-0686 (Electronic) 0965-8211 (Linking). https://doi.org/10.1080/09658211. 2013.800553. https://www.ncbi.nlm.nih.gov/pubmed/23721293

Smith, S. M. et al. (2018). Context specificity of automatic influences of memory. Journal of Experimental Psychology: Learning, Memory and Cognition (in press).

Storm, B. C., \& Angello, G. (2010). Overcoming fixation. Creative problem solving and retrieval-induced forgetting. Psychological Science, 21(9), 1263-1265. ISSN: 0956-7976. https://doi.org/10. 1177/0956797610379864. https://www.ncbi.nlm.nih.gov/pubmed/ 20693427

Storm, B. C., Angello, G., \& Bjork, E. L. (2011). Thinking can cause forgetting: Memory dynamics in creative problem solving. Journal of Experimental Psychology: Learning, Memory, and Cognition, 37(5), 1287-1293. ISSN: 1939-1285. https://doi.org/10.1037/a00 23921. https://www.ncbi.nlm.nih.gov/pubmed/21707211

Storm, B. C., \& Koppel, R. H. (2012). Testing the cue dependence of problem-solving-induced forgetting. The Journal of Problem Solving, 4(2). ISSN: 1932-6246. https://doi.org/10.7771/1932-6246. 1125

Vasconcelos, L. A. et al. (2017). To copy or not to copy: The influence of instructions in design fixation experiments. In Gero,
J. S. (Ed.) Design Computing and Cognition '16 (pp. 359375). Cham: Springer International Publishing. ISBN: 978-3-31944989-0. https://doi.org/10.1007/978-3-319-44989-0_20

Vul, E., \& Pashler, H. (2007). Incubation benefits only after people have been misdirected. Memory \& Cognition, 35(4), 701-710. ISSN: 0090-502X. https://doi.org/10.3758/bf03193308

Ward, T. B. (1994). Structured imagination: The role of category structure in exemplar generation. Cognitive Psychology, 27 (1), 1-40. ISSN: 00100285. https://doi.org/10.1037/e665402011365

Weldon, M. S., \& Bellinger, K. D. (1997). Collective memory: Collaborative and individual processes in remembering. Journal of Experimental Psychology: Learning, Memory, and Cognition, 23, 1160-1175. ISSN: 0278-7393. https://doi.org/10.1037/0278-7393. 23.5.1160

Wertheimer, M. (1945). Productive thinking. Oxford: Harper.

Wiley, J. (1998). Expertise as mental set: The effects of domain knowledge in creative problem solving. Memory \& Cognition, 26(4), 716-730. ISSN: 0090-502X. https://doi.org/10.3758/bf03211392

Wood, G., \& Pennington, J. (1973). Encoding and retrieval from long-term storage. Journal of Experimental Psychology, 99(2), 243-254. ISSN: 0022-1015. https://doi.org/10.1037/h0034636 